Hardy-Ramanujan Journal

Vol.18 (1995) 10-22

\title{
ON RIEMANN ZETA-FUNCTION AND ALLIED QUESTIONS-II
}

\section{BY}

\section{R. BALASUBRAMANIAN AND K. RAMACHANDRA}

§ 1. INTRODUCTION. It is best to begin with two definitions. We write $s=\sigma+i t$ unless otherwise stated.

DEFINITION 1. Let $\left\{a_{n}\right\}(n=1,2,3, \cdots)$ with $a_{1}=1$ be any sequence of complex numbers ( $a_{n}$ may depend on two parameters $T$ and $H$ with $T \geq H \geq$ a large positive constant to appear later) which vanish for all but finitely many $n$. Then $F_{0}(s)=\sum_{n=1}^{\infty} a_{n} n^{-s}$ is called a Titchmarsh polynomial.

DEEINITION 2. Let $\left.\left\{a_{n}\right\}(n=1,2,3, \cdots)\right)$ with $a_{1}=1$ be any sequence of complex numbers ( $a_{n}$ may depend on two parameters $T$ and $H$, with $T \geq H \geq a$ large positive constant, to follow). Suppose $F(s)=\sum_{n=1}^{\infty} a_{n} n^{-s}$ is convergent somewhere in the complex plane (depending on $T$ and $H$ to follow) and can be continued analytically in $\left(\sigma>0, T-H_{1} \leq t \leq T+H+H_{1}\right)$, where $H_{1}\left(\leq \frac{1}{2} T\right)$ is a suitable function of $T$, and there $|F(s)| \leq T^{A} \log H$ where $A \geq 1$ is any suitable constant. Then $F(s)$ is called an infinite Titchmarsh polynomial.

REMARK. In definitions 1 and 2 we may replace $n^{-*}(n \geq 1)$ by $\lambda_{n}^{-*}$ where 
$\left\{\lambda_{n}\right\}$ is any sequence of real numbers with $\lambda_{1}=1$ and $\frac{1}{A} \leq \lambda_{n+1}-\lambda_{n} \leq$ $A(n=1,2,3, \cdots), A \geq 1$ being $a$ constant. We are thus led to "Generalised Titchmarsh polynomial" and "Infinite generalised Titchmarsh polynomial".

CONJECTURE 1. There exist absolute numerical constants $c_{1}>0$ and $c_{2}>0$ such that for any Titchmarsh polynomial $F_{0}(s)$, we have

$$
\frac{1}{H} \int_{T}^{T+H}\left|F_{0}(i t)\right|^{2} d t \geq c_{1} \sum_{n \leq c_{2} H}\left|a_{n}\right|^{2} .
$$

CONJECTURE 2. There exist constants $c_{1}>0$ and $c_{2}>0$ (which may depend at most on A) such that for any infinite Titchmarsh polynomial $F(s)$, we have

$$
\frac{1}{H} \int_{T}^{T+H}|F(i t)|^{2} d t \geq \frac{1}{2} c_{1} \sum_{n \leq c_{2} H}\left|a_{n}\right|^{2}
$$

REMARK 1. By LHS of (2) we mean the limit of the mean value of $|F(s)|^{2}$ as $\sigma \rightarrow 0$ from the right. We do not know how to prove Conjecture 1. We believe that it implies the stronger Conjecture 2. But we cannot prove this implication.

REMARK 2. For many important applications it suffices to suppose that, in Conjectures 1 and $2,\left|a_{n}\right| \leq(n H)^{A}$, where $A \geq 1$ is some constant. But we cannot prove these conjectures nor the implication referred to in Remark 1 above even under this restriction.

We first prove (in § 3 ) the following theorem.

THEOREM 1. If $H_{1}>0$ is a large positive constant times $\log \log T$ and the infinite Titchmarsh polynomial $F(s)$ converges absolutely at Re $s=2$ and can be continued analytically in $\left(\sigma \geq-\delta, T-H_{1} \leq t \leq T+H+H_{1}\right)$ and there maximum of $|F(s)| \leq T^{A} \log H$, then Conjecture 1 implies

$$
\frac{1}{H} \int_{T}^{T+H}|F(i t)|^{2} d t \geq \frac{1}{2} c_{1} \sum_{n \leq c_{2} H}\left|a_{n}\right|^{2} .
$$

REMARK. The condition Re $s=2$ is unimportant. We can work with any constant in place of 2 . 
Next (in $\S 4$ and $\S 5$ ) we prove the following theorem. We define $d_{k}(n)$ (for complex $k$ ) by $(\zeta(s))^{k}=\sum_{n=1}^{\infty} d_{k}(n) n^{-s}$, Re $s \geq 2$.

THEOREM 2. Let $T$ and $H$ exceed large positive constants and let $k$ be any complex number with $|\boldsymbol{k}| \leq \log H$ and Re $k \geq 0$. Then conjecture 1 implies

$$
\frac{1}{H} \int_{T}^{T+H}\left|\left(\zeta\left(\frac{1}{2}+i t\right)\right)^{k}\right|^{2} d t \geq \frac{1}{2} c_{1} \sum_{n \leq c_{2} H}\left|d_{k}(n)\right|^{2} n^{-1} .
$$

Here we have assumed that $\zeta(s) \neq 0$ in $\left(\sigma>\frac{1}{2}, T-H_{1} \leq t \leq T+H+H_{1}\right)$ where $H_{1}$ is a large positive constant multiple of $\log T \log H(\log \log T)^{-1}$.

REMARK 1. We are unable, so far, to put Theorem 2 in the language mean square of infinite Titchmarsh polynomials. Also we believe that in Theorem 2 we can manage to take $H_{1}$ to be a positive constant multiple of $\log \log T$. We have been unable to prove this.

REMARK 2. Theorem 2 goes through for the zeta and $L$-functions of algebraic number fields (in place of $\zeta(s)$ ). In fact it has analogues for $D(s)$ (to be introduced in $\S 6$, and there with $\lambda_{n}=n$ ) in place of $\zeta(s$ ). For example we can select $\alpha=\frac{1}{2}$.

NOTATION. We use $c_{0}, c_{0}^{\prime}, c_{1}, c_{2}, \cdots$ to denote positive constants which depend at most on $A$ whenever it occurs. The $O$ symbols used extensively by Hardy and Littlewood and the symbols $\gg$ and $\ll$ of I.M. Vinogradov have the usual meaning. Sometimes we indicate the constant parameters on which these symbols imply by writing them below these symbols.

§ 2. APPLICATIONS OF THEOREMS 1 AND 2. It is not hard to deduce the following two Theorems 3 and 4 as corollaries to Theorems 1 and 2 respectively. (We concentrate on results mainly with the condition that $H$ does not exceed a large positive constant times $\log \log T$. We have dealt with the case $H$ exceeding a large positive constant times $\log \log T$ very satisfactorily elsewhere (without the help of Conjectures 1 or 2 ) in our previous papers (see the paper $\mathrm{I}^{[4]}$ of this series of papers for references). 
THEOREM 3. Conjecture 1 implies

$$
\frac{1}{H} \int_{T}^{T+H}\left|\zeta\left(\frac{1}{2}+i t\right)\right|^{2 k} d t \geq \frac{1}{2} c_{1} \sum_{n \leq c_{2} H}\left(d_{k}(n)\right)^{2} n^{-1}
$$

for all integers $k \geq 1$ and so we have first that LHS of $(5)$ is $\gg_{k}(\log H)^{k^{2}}$ and next

$$
\max _{T \leq t \leq T+H}\left|\zeta\left(\frac{1}{2}+i t\right)\right|>\operatorname{Exp}\left(\frac{3}{4} \sqrt{\frac{\log H}{\log \log H}}\right) .
$$

THEOREM 4. Pui $k=k_{0} e^{i \theta}$ where $\cos \theta \geq 0$ and $k_{0} \geq 1$ is any integer. Then under the conditions of Theorem 2, LHS of (4) is $\gg_{k}(\log H)^{\left|k^{2}\right|}$ and next with $z=e^{i \theta}$ we have

$$
\max _{T \leq t \leq T+H}\left|\left(\zeta\left(\frac{1}{2}+i t\right)\right)^{z}\right|>\operatorname{Exp}\left(\frac{3}{4} \sqrt{\frac{\log H}{\log \log H}}\right),
$$

LHS trivially $\infty$ if $\cos \theta<0$, of course on $\mathrm{RH}$.

REMARK 1. It is well-known that for complex $k$ and all $x \geq 2$, we have $\sum_{n \leq x}\left|d_{k}(n)\right|^{2} n^{-1} \gg_{k}(\log x)^{\left|k^{2}\right|}$. The inequalities $(6)$ and (7) with some positive constant $c_{0}$ in place of $\frac{3}{4}$ follow from (5) and (4). All that we have to prove is that for all $x$ exceeding a large positive constant, we have

$$
\max _{|k| \leq \log x}\left(\sum_{n \leq x}\left|d_{k}(n)\right|^{2} n^{-1}\right)^{|2 k|^{-1}}>\operatorname{Exp}\left(\cos \sqrt{\frac{\log x}{\log \log x}}\right) .
$$

This is proved by us in [2]. But in [1] R. Balasubramanian has shown by an ingenious method that the logarithms of both sides of (8) are asymptotic, as $x \rightarrow \infty$, with $c_{0}=0.75 \cdots$.

REMARK 2. In Theorems 3 and 4 we have stated results on the critical line. But we can also state these for any $\sigma$ with $\frac{1}{2}<\sigma<1$. But the result corresponding to $(8)$ is

$$
\max _{|k| \leq \log x}\left(\sum_{n \leq x}\left|d_{k}(n)\right|^{2} n^{-2 \sigma}\right)^{|2 k|^{-1}}>E x p\left(c_{0}^{\prime} \frac{(\log x)^{1-\sigma}}{\log \log x}\right)
$$


(where $c_{0}^{\prime}>0$ is constant) and so the results although valid for short intervals are not so good as those of H.L. Montgomery [3] who treats only long intervals like $0 \leq t \leq T$. His method fails for short intervals. Actually in [2] we have proved that the logarithm of LHS of $(9)$ is $O\left((\log x)^{1-\sigma}(\log \log x)^{-1}\right)$.

REMARK 3. It may be noted that the maximums in (8) and (9) even though we do not impose $|k| \leq \log x$ are really attained in the range $|k| \leq \log x$ and precisely when $|k|$ exceeds a positive constant power of $\log x$ (see [2]). Hence for Theorems 3 and 4 Conjectures 1 and 2 with $c_{1}(\log H)^{-100}$ in place of $c_{1}$ are enough.

REMARK 4. On the line $\sigma=1$ there are excellent results (see [5]).

$\S 3$. PROOF OF THEOREM 1. Let $s=i t$ and let $t$ exceed a large positive constant. For $X \geq 1$ we define $A_{X}(s)$ by

$$
\begin{aligned}
& A_{X}(s)=\frac{1}{2 \pi i} \int_{2-i \infty}^{2+i \infty} F(s+w) X^{2} \operatorname{Exp}\left(\left(\sin \frac{w}{100}\right)^{2}\right) \frac{d w}{w} \\
& =\sum_{n=1}^{\infty} \frac{a_{n}}{n^{i t}} \Delta\left(\frac{X}{n}\right)
\end{aligned}
$$

where for real $\chi>0, \Delta(\chi)$ is defined by

$$
\Delta(\chi)=\frac{1}{2 \pi i} \int_{2-i \infty}^{2+i \infty} \chi^{w} E_{x p}\left(\left(\sin \frac{w}{100}\right)^{2}\right) \frac{d w}{w} .
$$

Here $w=u+i v$ is a complex variable and $\left|\chi^{w}\right|=\chi^{u}$. By moving the line of integration to $u=4$ and $u=-4$ respectively we have

$$
\Delta(\chi)=O\left(\chi^{4}\right)=1+O\left(\chi^{-4}\right)
$$

and so the contribution to $A_{X}(s)$ from very large $n$ is very small. Notice that our condition on $a_{n}$ in definition 2 implies that $\left|a_{n}\right|$ does not exceed a fixed power (depending on $T$ and $H$ ) of $n$. Hence the expression (11) is a Titchmarsh polynomial plus a term whose absolute value is very small. We truncate the integral for $A_{X}(s)$ suitably and we have (with $Y \leq T^{\frac{1}{2}}$ )

$$
A_{X}(s)=\frac{1}{2 \pi i} \int_{u=2,|v| \leq Y} \cdots+O\left(T^{2 A \log H} X^{2}\left(E x p E x p\left(\frac{Y}{400}\right)\right)^{-1}\right) .
$$


Here moving the line of integration to $u=-\delta$, we have,

$$
A_{X}(s)=F(s)+O\left(T^{2 A \log H} X^{2}(\cdots)^{-1}\right)+O\left(\int_{u=-\delta} T^{2 A \log H} X^{-\delta} \frac{|d w|}{\left|w^{2}\right|}\right) .
$$

We note that $H$ is not more than a positive constant times $\log \log T$ and 80 by first choosing $X$ to be a very large positive constant power of $T^{A} \log H$ and then $Y$ to be a large positive constant times $\log \log T$, we see that

$$
A_{X}(s)=F(s)+\text { a very small term }
$$

Thus on using Conjecture 1 we have

$$
\frac{1}{H} \int_{T}^{T+H}|F(i t)|^{2} d t \geq \frac{3}{4} H^{-1} \int_{T}^{T+H}\left|A_{X}(s)\right|^{2} d t \geq \frac{1}{2} c_{1} \sum_{n \leq c_{2} H}\left|a_{n}\right|^{2}
$$

This proves Theorem 1 completely.

§ 4. FIRST PART OF THE PROOF OF THEOREM 2. Let now $s=\frac{1}{2}+\delta+i t$ ( $t>0$ large, $\delta>0$ now not a constant) and $X \geq 1$. With $B_{X}(s)$ now defined by

$$
\begin{gathered}
B_{X}(s)=\frac{1}{2 \pi i} \int_{2-i \infty}^{2+i \infty}(\zeta(s+w))^{k} X^{w} \operatorname{Exp}\left(\left(\sin \frac{w}{100}\right)^{2}\right) \frac{d w}{w} \\
=\sum_{n=1}^{\infty} \frac{d_{k}(n)}{n^{s}} \Delta\left(\frac{X}{n}\right),
\end{gathered}
$$

We have as before

$$
\Delta(\chi)=\frac{1}{2 \pi i} \int_{2-i \infty}^{2+i \infty} \chi^{w} \operatorname{Exp}\left(\left(\sin \frac{w}{100}\right)^{2}\right) \frac{d w}{w},(\chi>0)
$$

and so

$$
\Delta(\chi)=O\left(\chi^{4}\right)=1+O\left(x^{-4}\right) .
$$

Under the conditions of Theorem 2 we have for

$$
z=\sigma+i \tau,\left(\sigma>\frac{1}{2},|t-\tau| \leq c_{3} \log \log \log T\right)
$$




$$
\begin{gathered}
T-H_{1}+c_{3} \log \log \log T \leq \tau \leq T+H+H_{1}-c_{3} \log \log \log T \\
-c_{4} \frac{\log T}{\log \log T} \max \left(1, \log \left(\frac{c_{5}}{\left(\sigma-\frac{1}{2}\right) \log \log T}\right)\right) \leq \log |\zeta(x)| \leq c_{6} \frac{\log T}{\log \log T}
\end{gathered}
$$

and

$$
|\arg \zeta(z)| \leq c_{7} \frac{\log T}{\log \log T}
$$

For these results see [8] and also [6]. These results are due to $K$. Ramachandra and A. Sankaranarayanan. For convenient reference we state in \$ 6 a very general result of theirs for use by others for further work if necessary. Note that if $k=k_{1}+i k_{2}\left(k_{1}, k_{2}\right.$ real and $\left.k_{1} \geq 0\right)$ we have

$$
\begin{aligned}
\mid \zeta(z))^{k} \mid & =\left|\operatorname{Exp}\left(\left(k_{1}+i k_{2}\right)(\log |\zeta(z)|+i \arg \zeta(z))\right\rangle\right| \\
& =|-\zeta(z)| k_{1} \operatorname{Exp}\left(-k_{2} \arg \zeta(z)\right) \\
& \leq \operatorname{Exp}\left(c_{8} \frac{\log T \log H}{\log \log T}\right)
\end{aligned}
$$

As before we move the line of integration to $u=-\delta$ and we have

$$
B_{X}(s)=(\zeta(s))^{k}+O\left(T^{\frac{c g \log H}{\log \log T}} X^{2}(\cdots)^{-1}\right)+O\left(\int_{u=-\delta,|v| \leq Y} T^{\frac{\operatorname{col} \log H}{\log \log T}} X^{-\delta} \frac{|d w|}{|w|}\right)
$$

We note that $H$ is not more than a constant times $\log \log T$ and so by first choosing $X$ by

$$
X^{\frac{6}{2}}=T^{\frac{c 9 \log H}{\log \log T}}
$$

and then choosing $Y$ such that

$$
T^{\frac{\operatorname{col} \log H}{\log \log T}} \cdot T^{\frac{4}{6}} \frac{c_{\operatorname{cog}} \log H}{\log \log T}\left(\operatorname{Exp} E x p \frac{Y}{100}\right)^{-1}
$$

is very small we find that

$$
B_{X}(s)=(\zeta(s))^{k}+\text { a small quantity }+O\left(X^{-\frac{6}{2}}\left(\log \frac{1}{\delta}+\log Y\right)\right) .
$$

The last $O$-term in (29) is small if $T^{-\frac{D g}{\log \log T} T}\left(\log \frac{1}{\delta}+\log T\right)$ is small since $1 \leq Y \leq T^{\frac{1}{2}}$. In the next section we show that we can shoose $\delta$ to be a large 
positive constant times $T^{-\frac{\log H}{\operatorname{loglog} T}}$ in order to prove Theorem 2. Thus we have to choose $Y$ to be greater than a large positive constant multiple of

$$
\log \log \left(T^{\frac{8}{\delta} \operatorname{cg} \frac{\log H}{\log \log T}}\right)=\log \left(\frac{8}{\delta} \operatorname{cg} \frac{\log H \log T}{\log \log T}\right) .
$$

Thus (with $s=\frac{1}{2}+\delta+i t$ ) we have

$$
\begin{aligned}
\frac{1}{H} \int_{T}^{T+H}\left|(\zeta(s))^{k}\right|^{2} d t & \gg \frac{99}{100} c_{1} \sum_{n \leq c_{2} H}\left|d_{k}(n)\right|^{2} n^{-1-2 \delta} \\
& \geq \frac{3}{4} c_{1} \sum_{n \leq c_{2} H}\left|d_{k}(n)\right|^{2} n^{-1}
\end{aligned}
$$

§ 5. DIFFERENCE OF TWO INTEGRALS. The rest of the work consists in showing that $D$ defined by

$$
D=\frac{1}{H} \int_{T}^{T+H}\left(\left|\left(\zeta\left(\frac{1}{2}+i t\right)\right)^{k}\right|^{2}-\left|\left(\zeta\left(\frac{1}{2}+\delta+i t\right)\right)^{k}\right|^{2}\right) d t,
$$

has a small absolute value provided $\delta$ is small, but not too small since we want $\log \frac{1}{\delta}$ to be small. This would prove that

$$
\frac{1}{H} \int_{T}^{T+H}\left|\left(\zeta\left(\frac{1}{2}+i t\right)\right)^{k}\right|^{2} d t \geq \frac{1}{2} c_{1} \sum_{n \leq c_{2} H}\left|d_{k}(n)\right|^{2} n^{-1},
$$

which is precisely Theorem 2 .

By (24) we have

$$
\begin{aligned}
|D| & \leq \frac{1}{H} \int_{T}^{T+H}\left|\int_{\frac{1}{2}}^{\frac{1}{2}+\delta} \frac{\partial}{\partial \sigma}(\zeta(\sigma+i t))^{2 k} d \sigma\right| d t \\
& \leq \frac{2 k \mid}{H} \operatorname{Exp}\left(2 c_{8} \frac{\log T \log H}{\log \log T}\right) \int_{T}^{T+H} \int_{\frac{1}{2}}^{\frac{1}{2}+\delta}\left|\frac{\zeta^{\prime}(\sigma+i t)}{\zeta(\sigma+i t)}\right| d \sigma d t .
\end{aligned}
$$

To estimate the double integral in (33) we need a few lemmas. We begin with

LEMMA 1. Let $f(z)$ be analytic in $\left|z-z_{0}\right| \leq R$ and on the boundary of this disc let Re $f(z) \leq U$. Then

$$
f^{\prime}\left(z_{0}\right)=O\left(\left(U+\mid f\left(z_{0}\right) !\right) R^{-1}\right)
$$


where the implied constant is absolute.

PROOF. The lemma is well-known (see page 8, Theorem 2:4.1 of [7]).

LEMMA 2. We have, with $s=\sigma+i$ as in (33),

$$
\frac{\zeta^{\prime}(\sigma+i t)}{\zeta(\sigma+i t)}=O\left(\log T+|\log \zeta(s)|+\sum_{\rho \in D_{0}}|\rho-s|^{-1}\right)
$$

where $\rho$ runs over the zeros in the disc $D_{0}$ given by $|z-s| \leq R=\frac{1}{100}$.

PROOF. Put $z_{0}=s$ and

$$
f_{0}(z)=\frac{\zeta(z)}{\prod_{\rho \in D_{0}}\left(1-\frac{z-s}{\rho-s}\right)}
$$

we have

$$
\max _{z \in D_{0}}\left|f_{0}(z)\right| \leq \max _{x \in D_{1}}\left|f_{0}(z)\right| \text {. }
$$

where $D_{1}$ is the disc $|z-s| \leq \frac{1}{4}$. Hence by applying Lemma 1 to $f(z)=$ $\log f_{o}(z)$ we obtain the lemma.

LEMMA 3. We have

$$
\frac{1}{H} \int_{T}^{T+H} \int_{\frac{1}{2}}^{\frac{1}{2}+\delta}(\log T+|\arg \zeta(s)|) d \sigma d t=O(\delta \log T) .
$$

PROOF. Follows from the result of Ramachandra and Sankaranarayanan mentioned in (23).

LEMMA 4. We have

$$
\frac{1}{H} \int_{T}^{T+H} \int_{\frac{1}{2}}^{\frac{1}{2}+\delta} \max \left(1, \log \frac{c_{5}}{\left(\sigma-\frac{1}{2}\right) \log \log T}\right) d \sigma d t=O\left(\delta \log \log T+\delta^{\frac{1}{2}}\right) .
$$

PROOF. Follows from

$$
\int_{\frac{1}{2}}^{\frac{1}{2}+\delta} \log \left(\frac{1}{\sigma-\frac{1}{2}}\right) d \sigma \leq \sqrt{\delta}\left(\int_{\frac{1}{2}}^{\frac{1}{2}+\delta}\left(\log \frac{1}{\sigma-\frac{1}{2}}\right)^{2} d \sigma\right)^{\frac{1}{2}}
$$


LEMMA 5. We have

$$
\frac{1}{H} \int_{T}^{T+H} \int_{\frac{1}{2}}^{\frac{1}{2}+\delta}|\log | \zeta(\delta)|| d \sigma d t=O\left(\left(\delta \log \log T+\delta^{\frac{1}{2}}\right) \frac{\log T}{\log \log T}\right) .
$$

PROOF'. Follows from Lemma 4 and the result of Ramachandra and Sankaranarayanan mentioned in (23).

LEMMA 6. Let $\rho$ be a zero of the Riemann zeta-function with Re $\rho \leq \frac{1}{2}$. Then

$$
\frac{1}{H} \int_{T}^{T+H} \cdot \int_{\frac{1}{2}}^{\frac{1}{2}+\delta} \frac{d \sigma d t}{|\rho-s|}=O\left(\delta^{\frac{1}{2}} \log T\right)
$$

PROOF. Let $\rho=\beta+$ ir with $\beta \leq \frac{1}{2}$. Then $|\rho-s|^{-1} \leq\left|\frac{1}{2}-s+i \gamma\right|^{-1}$ and hence

$$
\begin{gathered}
\int_{T}^{T+H}\left|\frac{1}{2}-\sigma-i t+i \gamma\right|^{-1} d t=\int_{|t-\gamma| \leq \alpha-\frac{1}{2}} \cdots+\int_{T \geq|t-\gamma| \geq \sigma-\frac{1}{2}} \cdots \\
=O(1)+O(\log T)+O\left(\log \frac{1}{c-\frac{1}{2}}\right)
\end{gathered}
$$

and hence the lemma followg.

LEMMA 7. The number of zeros $\rho$ counted with $i \leq \operatorname{Im} \rho \leq T+1$ and $0 \leq \operatorname{Im} \rho \leq 1$, is $O(\log T)$.

PROOF. This is well-known. See for example Theoren 3.1.1 on page 11 of $[7]$.

LEMMA 8. We have

$$
\frac{1}{H} \int_{T}^{T+H} \int_{\frac{1}{2}}^{\frac{1}{2}+\delta}\left(\sum_{\rho \in D_{0}}|\rho-s|^{-1}\right) d \sigma d t=O\left(\delta^{\frac{1}{3}}(\log T)^{3}\right)
$$

PROOF. Of course the sum over $\rho$ depends on $s$. But we extend the sum over all the zeros of $\zeta(s)$ in $T-1 \leq t \leq T+H+1$. Now we can interchange the sum over $\rho$ and the double integral. The number of zeros is now $O(B \log T)$. The lemma now follows from Lemma 6. 
LEMMA 8. We have

$$
D=O\left(\delta^{\frac{1}{2}}(\log T)^{4} E x p\left(2 c_{8} \frac{\log T \log H}{\log \log T}\right)\right)
$$

PROOF. Follows from (33) and Lemmas 2 to 7.

LEMMA 9. $|D|$ is very small if $\delta$ is taken to be a large positive constani power of $\operatorname{Exp}\left(-\frac{\log T \log H}{\log \log T}\right)$.

PROOF. Follows from Lemma 8.

Theorem 2 now follows from the results of $\S 4$ and $\S 5$.

\section{§ 6. A RESULT OF K. RAMACHANDRA AND A. SANKARA-} NARAYANAN. While stating Lemma 1 of $\S 2$ on page 392 of [6] the condition (4) of that paper is not necessary. It was meant for other purposes. Accordingly we state this once again.

THEOREM 5. (K. RAMACHANDRA AND A. SANKARANARAXANAN), Let

$$
D(s)=\sum_{n=1}^{\infty} a_{n} \lambda_{n}^{-s}
$$

where $a_{1}=1=\lambda_{1}, \frac{1}{A} \leq \lambda_{n+1}-\lambda_{n i} \leq A(A \geq 1$ is a constant $)\left\{\lambda_{n}\right\}$ is any sequence of real numbers and $\left\{a_{n}\right\}$ is any sequence of complex numbers with $\left|a_{n}\right| \leq n^{A}$. Let $\alpha>\delta(\delta>0$ a constani $)$ and let $R(H, \alpha)$ denote the rectangle $\left(\sigma \geq \alpha, T_{1}-H \leq t \leq T_{1}+H\right)$. Let $D(s)$ be continuable analytically in $R(H, \alpha-\delta)$ and there $\max |D(s)| \leq T^{A}$ (where $A_{5} \log \log \log T \leq H \leq \frac{1}{2} T$ ) and $T_{1}$ is any number lying between $T-H$ and $2 T+H$. Let $D(s) \neq 0$ in $R(H, \alpha)$. Then for $t=T_{1}, s=\sigma+i t$ in $R(H, \alpha)$ we have uniformily for $\sigma \geq \alpha, t=T_{1}$

$$
-A_{1} \frac{\log T}{\log \log T} \max \left[1, \log \left(\frac{A_{2}}{(\sigma-\alpha) \log \log T}\right)\right] \leq \log |D(s)| \leq A_{3} \frac{\log T}{\log \log T}
$$

and

$$
|\arg D(s)| \leq A_{4} \frac{\log T}{\log \log T}
$$

Here $A_{1}, A_{2}, \cdots, A_{5}$ are positive constants depending only on $\delta$ and $A$.

Note. It is enough to assume $D(s) \neq 0$ in $\left(\sigma>\alpha, T_{1}-H \leq t \leq T_{1}+H\right)$. 


\section{REFERENCES}

[1] R. BALASUBRAMANLAN, On the frequency of Titchmarsh's phenomenon for $\zeta(s)$-IV, Hardy-Ramanujan J., 9 (1986), 1-10.

[2] R. BALASUBRAMANIAN AND K. RAMACHANDRA, On the frequency of Titchmarsh's phenomenon for $\zeta(s)$-III, Proc. Indian Acad. Sci., (Section A), 86 (1977)), 341-351.

[3] H.L. MONTGOMERY, Extreme values of the Riemann zeta-function, Comment. Math. Helv., 52 (1977), 511-518.

[4] K. RAMACHANDRA, On Riemann zeta-function and allied questionsI, Asterisque, vol. 209 (1992), 57-72.

[5] K. RAMACHANDRA; On the frequency of Titchmarsh's phenomenon for $\zeta(s)$-IX, Hardy-Ramanujan J., 13 (1990), 28-33.

[6] K. RAMACHANDRA, On the zeros of a class of generalised Dirichlet series-VII, Annales Acad. Sci. Fenn., Ser AI Math., 14 (1989), 27-40.

[7] K. RAMACHANDRA, Riemann zeta-function, Ramanujan Institute, Madras University, Madras (1979), 16pp.

[8] K. RAMACHANDRA AND A. SANKARANARAYANAN, On some theorems of Littlewood and Selberg-III, Annales Acad. Sci. Fenn., Ser Al Math., 16 (1991), 139-149.

ADDRESS OF THE AUTHORS :

R. BALASUBRAMANIAN

MATSCIENCE

THARAMANI P.O.

MADRAS 600113

TAMIL NADU, INDIA 
K. RAMACHANDRA

SCHOOL OF MATHEMATICS

TATA INSTITUTE OF FUNDAMENTAL RESEARCH HOMI BHABHA ROAD

COLABA

BOMBAY 400 005, INDIA

MANUSCRIPT COMPLETED ON 12 JUNE 1994. 